Internat. J. Math. \& Math. Sci.

Vol. 24, No. 5 (2000) 305-313

S0161171200000910

(c) Hindawi Publishing Corp.

\title{
UNIFORMLY CONVERGENT SCHEMES FOR SINGULARLY PERTURBED DIFFERENTIAL EQUATIONS BASED ON COLLOCATION METHODS
}

\author{
DIALLA KONATE
}

(Received 29 May 1998)

\begin{abstract}
It is well known that a polynomial-based approximation scheme applied to a singularly perturbed equation is not uniformly convergent over the geometric domain of study. Such scheme results in a numerical solution, say $\sigma$ which suffers from severe inaccuracies particularly in the boundary layer. What we say in the current paper is this: when one uses a grid which is not "too coarse" the resulted solution, even being nonuniformly convergent may be used in an iterated scheme to get a "good" approximation solution that is uniformly convergent over the whole geometric domain of study.

In this paper, we use the collocation method as model of polynomial approximation. We start from a precise localization of the boundary layer then we decompose the domain of study, say $\Omega$ into the boundary layer, say $\Omega_{\epsilon}$ and its complementary $\Omega_{0}$. Next we go to the heart of our work which is to make a repeated use of the collocation method. We show that the second generation of polynomial approximation is convergent and it yields an improved error bound compared to those usually appearing in the literature.
\end{abstract}

Keywords and phrases. Singular perturbation, stiff equation, boundary layer, polynomial approximation, collocation method, domain decomposition.

2000 Mathematics Subject Classification. Primary 34D15, 65C20, 65M70, 65L06.

1. Introduction. We consider the following family of singularly perturbed initial value problems (SPIVP) also known as diffusion-convection problems (cf. Gartland $[4,5])$ :

$$
\begin{gathered}
L_{\epsilon} u \equiv \epsilon u^{(m)}+\sum_{i=0}^{m-1} a_{i} u^{(i)}=f, \quad a \leq x \leq b(a<b), \\
u^{(i)}(a)=\gamma_{i}, \quad \gamma_{i} \in \mathbb{R}, 0 \leq i \leq m-2, \\
u^{(m-1)}(a)=\gamma_{m-1}, \quad \gamma_{m-1}=\gamma_{m-1} \in \in \mathbb{R}, \\
\gamma_{m-1}=c_{-1} \epsilon^{-1}+c_{0} .
\end{gathered}
$$

Let us recall that this family of SPIVP is used to describe processes that arise from such various areas as biochemical kinetics, plasma physics, mechanical and electrical systems.

$\mathbb{C}^{m}(\Omega)$ denotes the space consisting of the functions which, together with their derivatives up to order $m$ are continuous. $\mathbb{C}^{m}(\Omega)$ is equipped with the maximum norm which reads

$$
\|u\|_{m, \infty}=\max _{k} \sup _{x \in \bar{\Omega}}\left|\frac{\partial_{k}}{\partial x^{k}}(x)\right| .
$$


If the bound on $\left|D^{k} u(x)\right|$ is taken over $\Omega_{\epsilon}$ (respectively, $\Omega_{0}$ ) then the correspondent maximum norm will be written $\|u\|_{m, \infty, \epsilon}$ (respectively, $\|u\|_{m, \infty, 0}$ ). When $m=0$ then the expression of the maximum norm simplifies over $\mathbb{C}^{m}(\Omega)$ in being $\|u\|_{\infty}$. Same way, if the domain of reference is $\Omega_{\epsilon}$ (respectively, $\Omega_{0}$ ) instead of $\Omega$, then the simplified expression for the maximum norm turns to be $\|u\|_{\infty, \epsilon}$ (respectively, $\|u\|_{\infty, 0}$ ).

We focus now on problem (1.1) which is stiff in the sense that its solution exhibits a boundary layer near the point $x=a$ under the basic hypothesis (1.3) that follows and that is valid throughout the paper. This basic hypothesis writes

$$
\begin{aligned}
& a_{i} \in L^{1}(\Omega), \quad 0 \leq i \leq m-1, \\
& a_{m-1}(x) \geq \alpha>0, \quad x \in \bar{\Omega} .
\end{aligned}
$$

It is well known from the classical papers from Lucas and Reddien [7] or de Boor and Swartz [3] that if $u \in \mathbb{C}^{n}(\Omega)$ is the solution of an initial value problem (IVP) of order $m(m \leq n)$, then its collocation approximation, say, $\sigma$ with respect to a uniform grid constructed with a stepsize $h$ is such that

$$
\left\|u^{(j)}-\sigma^{(j)}\right\|_{\infty} \leq h^{n-m} \cdot\left\|u^{(n)}\right\|_{\infty}, \quad 0 \leq j \leq m .
$$

From Gartland [5, Theorem 1.4, page 635], we know that $u$ solution of problem (1.1) is such that

$$
\left\|u^{(j)}-\sigma^{(j)}\right\|_{\infty} \leq C \cdot \epsilon^{m-2-j}, \quad j \geq 0
$$

thus if $n$ is taken such that $n=m+k$, then from inequality (1.4) we get

$$
\left\|u^{(j)}-\sigma^{(j)}\right\|_{\infty} \leq \epsilon^{-(2+k)} \cdot h^{k}, \quad 0 \leq j \leq m
$$

which implies that the collocation method is not uniformly convergent. It is also known that the numerical inaccuracies which appear in the boundary layer do not disappear with a mesh refinement. As reported in the literature, the intuitive expectation that the error should decrease with a mesh refinement falls down when the mesh size is taken of the same order of magnitude as the parameter $\epsilon$. To remedy that inconvenience, some authors have constructed exponentially fitted schemes or schemes that are based on special meshes (cf. Ascher [1], Ascher and Weiss [2], and Gartland [4, 5]). Hereafter, for the same goal, we do act in three steps that are:

(1) at first, we do locate precisely the boundary layer $\Omega_{\epsilon}$. This leads to decompose, in "natural way" $\Omega$ into $\Omega_{\epsilon}$ and $\Omega_{0}=\Omega-\Omega_{\epsilon}$,

(2) next, we apply two times the collocation method with respect to some specific grids,

(3) at the end, we do show that the second generated collocation approximation is uniformly convergent over the whole domain $\Omega$ and we show that the error is less than $H^{r+3}$ while the best error bounds in the literature are in $O\left(H^{r+1}\right)$.

2. Localization of the boundary layer. At first, we set the following definition.

DEFINITION 2.1. A regular function $\theta$ is said to be a corrector for problem (1.1) if $\left(Y_{q}+\theta\right)$ obeys the boundary conditions of problem (1.1) where $Y_{q}$ is an outer approximation to the solution $u$ of problem (1.1). 
Next we define what we call a threshold of acceptance as follows.

DEFINITION 2.2. We consider $\eta \in \mathbb{R}_{+}^{*}, \eta \ll \epsilon \cdot \eta$ is said to be a threshold of acceptance if:

$$
|x|=O(\eta) \text { then we set } x=0 \text {, for } x \in \mathbb{R} .
$$

We claim the following lemma.

LEMMA 2.3. The regular function (boundary layer function) $\theta$ defined by

$$
\theta(x)=(-1)^{m-1} \cdot \theta_{a} \cdot \epsilon^{m-2} \cdot(x-a)^{m-1} \cdot \exp \left(-(x-a) \cdot \epsilon^{-1}\right)
$$

is a corrector for problem (1.1), where

$$
\theta_{a}=\left(\gamma^{m-1}-\epsilon Y_{q}^{(m-1)}(a)\right) \cdot((m-1) !)^{-1}
$$

and $Y_{q}$ stands for a q th order regular asymptotic to $u$.

Proof. We begin by defining a regular (outer) asymptotic expansion $Y_{q}$ of order $q$ to $u$. To do so, let us first define some additional linear operators $L, L_{\epsilon}$, and $I_{\epsilon}$ by setting:

$$
\begin{gathered}
L u \equiv \sum_{i=0}^{m-1} a_{i} u^{(i)}, \\
L_{\epsilon} \equiv \epsilon u^{(m)}+\sum_{i=0}^{m-1} a_{i} u^{(i)}=\epsilon u^{(m)}+L u, \\
I_{\epsilon} u \equiv\left(I_{\epsilon, 0} u, I_{\epsilon, 1} u, \ldots, I_{\epsilon, m-1} u\right),
\end{gathered}
$$

where

$$
I_{\epsilon, i} u=u^{(i)}(a), \quad 0 \leq i \leq m-2, \quad I_{\epsilon, m-1} u=\epsilon u^{(m-1)}(a),
$$

then $Y_{q}$ is given by

$$
Y_{\mathcal{q}}=\sum_{i=-1}^{q} \epsilon^{i} y_{i},
$$

where the $q+2$ coefficient functions $y_{i}$ are defined by:

$$
\begin{array}{ll}
L y_{-1}=0, & I y_{-1}=0, \\
L y_{0}=f, & I y_{0}=\gamma=\left(\gamma_{0}, \ldots, \gamma_{m-2}\right), \\
L y_{i}=-y_{i-1}^{(m)}, & I y_{i}=0, \quad 1 \leq i \leq q,
\end{array}
$$

set

$$
v=u-Y_{q}
$$

it is well known that

$$
L_{\epsilon} v=L_{\epsilon} u-L_{\epsilon} Y_{q}=-\epsilon^{q+1} y_{q}^{(m)}
$$


by construction we have

$$
\begin{gathered}
v^{(i)}(a)=0 ; \quad 0 \leq i \leq m-2 \\
\epsilon v^{(m-1)}(a)=\epsilon u^{(m-1)}(a)-\epsilon Y_{q}^{(m-1)}(a)=\gamma_{m-1}-\epsilon \sum_{i=-1}^{q} \epsilon^{i} y_{i}^{(m-1)} .
\end{gathered}
$$

We have

$$
\begin{gathered}
\theta^{(i)}(a)=0, \quad \text { for } 0 \leq i \leq m-2, \\
\theta^{(m-1)}(a)=((m-1) !) \cdot \epsilon^{-1} \cdot \theta_{a}=\epsilon^{-1}\left(\gamma_{m-1}-\epsilon \cdot Y_{q}^{(m-1)}(a)\right) .
\end{gathered}
$$

So it is obvious from (2.10a) and (2.10b) that $w=v-\theta$ is such that

$$
w^{(i)}(a)=0 ; \quad 0 \leq i \leq m-2, \quad \epsilon w^{(m-1)}(a)=0 .
$$

The boundary layer being a neighbourhood of $x=a$, where the corrector (which is an exponentially decaying function) is significantly different from zero, we set the boundary layer to be that subset of $\Omega$, where $\exp \left(-(x-a) \cdot \epsilon^{-1}\right)$ is greater than the threshold of acceptance $\eta$. More precisely, we set the following definition.

DEFinition 2.4. For a given and fixed threshold of acceptance $\eta$, setting $r=-\epsilon^{-1}$, we define the boundary layer $\Omega_{\epsilon}$ to be

$$
\Omega_{\epsilon}=[x \in \Omega ; \exp (r(x-a))>\eta]=\left[x \in \Omega ;(x-a)<\epsilon \log \left(\eta^{-1}\right)\right]
$$

we set $\delta_{\epsilon}=\epsilon \log \left(\eta^{-1}\right)$ to be the length of the boundary layer.

We set

$$
\bar{x}_{\epsilon}=a+\delta_{\epsilon}
$$

and we have

$$
\left.\Omega_{\epsilon}=\right] a, \bar{x}_{\epsilon}[
$$

\section{The first polynomial approximation}

3.1. Construction of a grid. We begin with constructing a nonuniform grid as follows.

At first, we do realize a discretization of $\Omega_{\epsilon}$ with a stepsize $h$ such that

$$
h=\frac{\left(\bar{x}_{\epsilon}-a\right)}{N_{\epsilon}}=\frac{\delta_{\epsilon}}{N_{\epsilon}}, \quad N_{\epsilon} \in \mathbb{N}^{*} .
$$

Thus we obtain in $\Omega_{\epsilon} N_{\epsilon}+1$ grid points. We denote this grid $G_{h}$ which is determined by

$$
x_{0}=a, \quad x_{i}=a+i h, \quad 1 \leq i \leq N_{\epsilon}, \quad x_{N_{\epsilon}}=\bar{x}_{\epsilon} .
$$

Next we do realize a discretization of $\Omega_{0}$ with a stepsize $H$ such that

$$
H=\frac{\left(b-\bar{x}_{\epsilon}\right)}{N_{0}}, \quad N_{0} \in \mathbb{N}^{*} .
$$

We obtain a second grid, say, $G_{H}$. We constitute a nonuniform grid over $\Omega$, say, $G_{h, H}$ by merging $G_{h}$ and $G_{H}$. 
3.2. Construction of the approximation polynomial. In fact, we make use of the collocation method to construct the approximation polynomial. The collocation approximation to problem (1.1), say, $\sigma$ is defined as follows.

For each interval $\left.I^{i}=\right] x_{i} x_{i+1}$ [, where $x_{i}$ and $x_{i+1}$ are two knots of the grid $G_{H, h}$ both located within $G_{H}$ or $G_{h}$ at a time; we denote by $\sigma_{I_{i}}$ the restriction of $\sigma$ to $I_{i}$; i.e., $\sigma_{I_{i}}=\sigma / I_{i}$. For $r \geq m \sigma$ is defined by

$$
\sigma \in \mathscr{P}(m+r, m+r+1)=\left[v \in \mathbb{C}^{m+r}(\Omega), v_{I_{i}} \in P_{m+r}\right],
$$

$P_{m+r}$ denotes the set of polynomials of degree at most $m+r$. Thus we have

$$
\sigma_{I_{i}}(x)=\sum_{i=0}^{m+r} \alpha_{i j} x^{i}, \quad \alpha_{i j} \in \mathbb{R} .
$$

The coefficients $\alpha_{i j}(0 \leq j \leq m+r)$ are determined by solving the following linear system:

$$
\begin{aligned}
& L_{\epsilon} \sigma_{I_{i}}\left(t_{i j}\right)=f\left(t_{i j}\right), \quad 1 \leq j \leq r, \\
& \sigma_{I_{i}}^{(v)}\left(x_{i}\right)=\sigma_{I_{i-1}}^{(v)}\left(x_{i}\right), \quad 0 \leq v \leq m .
\end{aligned}
$$

Each $t_{i j}(1 \leq j \leq r)$ denotes a collocation point within $I_{i}$ if $u \in \mathbb{C}^{m+r+1}(\Omega)$. Then from (1.4), we extract the following relation:

$$
\left\|u^{(j)}-\sigma^{(j)}\right\|_{\infty} \leq C \cdot \Delta^{r+1} \cdot\left\|u^{(m+r+1)}\right\|_{\infty}, \quad 0 \leq j \leq m,
$$

where $C$ is a constant independent of $\epsilon$ and $\Delta$ is such that $\Delta=h \in \Omega_{\epsilon}$ and $\Delta=H \in \Omega_{0}$. The determination of $\sigma$ allows us to set up the following problem:

$$
\begin{aligned}
& L v=\sum_{i=0}^{m-1} a_{i} v^{i}=f-\epsilon \sigma^{(m)} \in \Omega, \\
& v^{i}(a)=\gamma_{i}, \quad 0 \leq i \leq m-2,
\end{aligned}
$$

where $\gamma_{i} \in \mathbb{R}$. We set

$$
w=u-v
$$

From now on we suppose that $h$ is taken such that

$$
h \leq K \cdot(\epsilon \cdot H)^{1+1 /(r+1)},
$$

where $K=\left(b-\bar{x}_{\epsilon}\right)^{-1} \cdot \log \left(\eta^{-1}\right)$. Inequality (3.10) may be written as follows:

$$
h^{r+1} \leq K^{r+1}(\epsilon \cdot H)^{r+2} .
$$

Now we are ready to claim the following lemma.

LEMmA 3.1. We assume that (3.10) holds true and $u, v, \in \mathbb{C}^{m+r+1}(\Omega)$ with $r \geq 1$. Then we have that 
(i) $\sigma$, the collocation solution to $u$, satisfies

$$
\sup _{x \in \Omega_{\epsilon}}\left|u^{(j)}-\sigma^{(j)}\right| \leq C \cdot \epsilon^{-1} \cdot H^{r+2}, \quad \sup _{x \in \Omega_{0}}\left|u^{(j)}-\sigma^{(j)}\right| \leq C \cdot H^{r+1},
$$

(ii) $w$ satisfies

$$
\|w\|_{\infty} \leq C \cdot \epsilon \cdot H^{r+1},
$$

where $C$ represents various constants that are independent of $\epsilon, h$, and $H$.

Proof. Applied to problem (1.1) considered under the hypotheses of Lemma 3.1; relation (3.7) results in (3.12). In addition, $w$ is such that

$$
\begin{gathered}
L w=\sum_{i=0}^{m-1} a_{i} w^{i}=-\epsilon\left(u^{(m)}-\sigma^{(m)}\right)=g \in \Omega, \\
w^{i}(a)=0, \quad 0 \leq i \leq m-2 .
\end{gathered}
$$

Using the Green's function, $w$ can be written as follows:

$$
w(x)=\int_{a}^{b} G(x, t) g(t) d t
$$

where

$$
g=\epsilon\left(u^{(m)}-\sigma^{(m)}\right)
$$

Then from (3.12) we pull that

$$
\begin{aligned}
& |g(x)| \leq C \cdot H^{r+2} \quad \text { for } x \in \Omega_{\epsilon}, \\
& |g(x)| \leq C \cdot \epsilon \cdot H^{r+1} \quad \text { for } x \in \Omega_{0} .
\end{aligned}
$$

Hence, from the equality

$$
w(x)=\int_{a}^{\bar{x}_{\epsilon}} G(x, t) g(t) d t+\int_{\bar{x}_{\epsilon}}^{b} G(x, t) g(t) d t
$$

and taking account of the continuity of function $G$, equation (3.18) leads to

$$
|w(x)| \leq C \cdot \epsilon \cdot H^{r+2}+C \cdot \epsilon \cdot H^{r+1}, \quad x \in \Omega,
$$

that is (3.13), where $C$ represents various constants independent of $\epsilon$. This ends the proof.

REMARK 3.2. $N_{0}$ represents the number of subintervals within the grid $G_{H}$ while $N_{\epsilon}$ is that within the grid $G_{h} . N_{0}$ and $N_{\epsilon}$ are such that if $r$ the number of collocation points goes to infinity, then $N_{\epsilon}$ tends to $N$ such that $N=N_{0}$.

Proof. $N_{\epsilon}=\delta \cdot h^{-1}$, where $h=K \cdot(\epsilon \cdot H)^{1+1 /(r+1)}$ and $K=\left(b-\bar{x}_{\epsilon}\right)^{-1} \cdot \log (\eta-1)$ then we get $N_{\epsilon}=\left(b-\bar{x}_{\epsilon}\right) \cdot H^{-1-1 /(r+1)}$; so, for $\epsilon$ fixed, when $r$ goes to infinity then $N_{\epsilon}$, obviously, goes to $N=\left(b-\bar{x}_{\epsilon}\right) \cdot H^{-1}$ that is $N_{0}$. 
4. The collocation method iterated. Within this section, we focus especially on $v$ solution of (3.7). Let us consider the collocation approximation to $v$, we construct as follows: we add $n_{\epsilon}$ (respectively, $n_{0}$ ) new collocation points to those of $G_{h}$ (respectively, $G_{H}$ ) to get a new grid $N G_{h}$ (respectively, $N G_{H}$ ). Next we merge these two grids to get one nonuniform grid, say, $N G_{h, H}$ covering all of $\Omega$. Let us denote $s$ the collocation approximation to $v$ with respect to the grid $N G_{h, H}$. We claim the following lemma.

LEMMA 4.1. For $\epsilon>0$ fixed, we assume that the hypotheses of Lemma 3.1 hold true. Then for the choices $n_{\epsilon}=r$ and $n_{0}=1$, the following estimates hold true for $0 \leq j \leq$ $m-1$,

$$
\sup _{x \in \Omega_{\epsilon}}\left|v^{(j)}-s^{(j)}\right| \leq C \cdot \epsilon \cdot H^{2(r+2)}, \quad \sup _{x \in \Omega_{0}}\left|v^{(j)}-s^{(j)}\right| \leq C \cdot H^{r+3} .
$$

Proof. Inequalities (4.1) are what (1.4) turns to be under the hypotheses of Lemma 4.1 and when $v$ is the solution of an $(m-1)$-order differential equation and $v$ is supposed to lie on $\mathbb{C}^{m+2 r+1}\left(\Omega_{\epsilon}\right)$ (respectively, $\mathbb{C}^{m+r+2} \Omega_{0}$ ).

All the preceding results constitute a pavement to the following statement.

THEOREM 4.2. For $\epsilon>0$ fixed, we suppose that $u$ and $v$ are sufficiently regular. Under hypothesis (3.10), the following estimate holds true:

$$
\left\|u^{(i)}-s^{(i)}\right\| \leq C \cdot \max \left(\epsilon \cdot H^{r+1}, H^{r+3}\right), \quad 0 \leq i \leq m-1,
$$

where $C$ stands for a constant independent of $\epsilon$.

PRoof. We start from the following obvious triangle inequality:

$$
\left\|u^{(i)}-s^{(i)}\right\|_{\infty} \leq\left\|u^{(i)}-v^{(i)}\right\|_{\infty}+\left\|v^{(i)}-s^{(i)}\right\|_{\infty} .
$$

As a consequence of (3.13), we have

$$
u^{(i)}(x)-v^{(i)}(x)=\epsilon \cdot H^{r+1} \cdot q^{(i)}(x), \quad 0 \leq i \leq m+r+1,
$$

which implies that

$$
\left\|u^{(i)}-v^{(i)}\right\|_{\infty} \leq C \cdot \epsilon \cdot H^{r+1}
$$

in addition Lemma 4.1 implies that

$$
\left\|v^{(i)}-s^{(i)}\right\|_{\infty} \leq C \cdot H^{r+3} .
$$

Altogether, relations (4.5) and (4.6) result in Theorem 4.2.

To end this paper, we have put our theoretical claims to the test by carrying out some interesting numerical experiments.

5. Numerical tests. As test problems, we consider a class of those that we draw from Kadalbajoo and Rao [6] which is well known to be reluctant to the classical numerical methods and which numerical treatment exhibits most of the troubles we 
have spoken of. This class is defined as follows:

$$
\begin{gathered}
\epsilon u^{\prime \prime}+u^{\prime}-u=0, \quad 0 \leq x \leq 1, \\
u(0)=\alpha, \\
u(1)=\beta
\end{gathered}
$$

for which the exact solution $u$ is given by

$$
u(x)=(\alpha-\beta \cdot \exp (-1)) \cdot \exp \left(-\frac{x}{\epsilon}\right)+\beta \cdot \exp (-1) \cdot \exp (x)+O(\epsilon) .
$$

All experiments displayed in Table 5.1 have confirmed the theoretical statements. Even our schemes seem to work when we take $N_{\epsilon}=N_{0}=N$ for $r$ enough large but not too much. For small values of $r$, the numerical results can be slightly altered. Such situation is under consideration in the experience numbered 2 which outputs are shown in Table 5.1.

Hereunder, the numbering coding and the denomination present as follows: $\mathrm{Nb}$ denotes the serial number of the numerical test, XEB measures the thickness of the boundary layer, NEPS denotes the number of the discretization points within $\Omega_{\epsilon}$ and $\mathrm{N}_{0}$ denotes those within $\Omega_{0}, E-q$ stands for $10^{q}$, GNEPS represents in percentage the gain in terms of error bound obtained by our scheme within $\Omega_{\epsilon}$ when passing from the first use of the collocation method to its second use, GNO is defined similarly to GNEPS with respect to $\Omega_{0}, v 1$ denotes the numerical approximation to $u$ generated by the first application of the collocation method and $v 2$ denotes the approximation solution obtained from its second application. We also use the following notations to display the outcomes of the numerical experiments:

$$
\|v\|_{\infty}=\max _{x \in \bar{\Omega}}|v(x)|, \quad\|v\|_{\infty, \epsilon}=\max _{x \in \bar{\Omega}_{\epsilon}}|v(x)|, \quad\|v\|_{\infty, 0}=\max _{x \in \bar{\Omega}_{0}}|v(x)|
$$

thus

$$
\begin{aligned}
\mathrm{GNEPS} & =\frac{\|u-v 1\|_{\infty, \epsilon}-\|u-v 2\|_{\infty, \epsilon}}{\|u-v 1\|_{\infty, \epsilon}} \cdot 100, \\
\mathrm{GNO} & =\frac{\|u-v 1\|_{\infty, \epsilon}-\|u-v 2\|_{\infty, 0}}{\|u-v 1\|_{\infty, 0}} \cdot 100 .
\end{aligned}
$$

The output of the numerical experiments are displayed in Table 5.1.

TABLE 5.1.

\begin{tabular}{ccccccc}
\hline $\mathrm{Nb}$ & XEB & EPS & NEPS & $\mathrm{N}_{0}$ & GNEPS & GNO \\
\hline 1 & $1.15 * E-2$ & $E-3$ & 10 & 3 & 53 & 28 \\
\hline 2 & $1.15 * E-2$ & $E-3$ & 10 & 10 & 53 & -0.92 \\
\hline 3 & $1.15 * E-3$ & $E-4$ & 60 & 10 & 19 & 19 \\
\hline 4 & $1.15 * E-3$ & $E-4$ & 20 & 10 & 58 & 57 \\
\hline 5 & $1.15 * E-4$ & $E-5$ & 30 & 10 & 38 & 38 \\
\hline 6 & $1.38 * E-4$ & $E-5$ & 120 & 100 & 12 & 12 \\
\hline 7 & $1.38 * E-5$ & $E-6$ & 60 & 20 & 23 & 23 \\
\hline
\end{tabular}




\section{REFERENCES}

[1] U. Ascher, Two families of symmetric difference schemes for singular perturbation problems, Numerical Boundary Value ODEs (Vancouver, B.C., 1984) (Boston, Mass.), Birkhauser Boston, 1985, pp. 173-191. MR 87h:65125. Zbl 568.65053.

[2] U. Ascher and R. Weiss, Collocation for singular perturbation problems. I. First order systems with constant coefficients, SIAM J. Numer. Anal. 20 (1983), no. 3, 537-557. MR 85a:65113. Zbl 523.65064.

[3] C. de Boor and B. Swartz, Collocation at Gaussian points, SIAM J. Numer. Anal. 10 (1973), 582-606. MR 51\#9528. Zbl 232.65065.

[4] E. C. Gartland, Jr., Uniform high-order difference schemes for a singularly perturbed twopoint boundary value problem, Math. Comp. 48 (1987), no. 178, 551-564, S5-S9. MR 89a:65116. Zbl 621.65088.

[5] _ Graded-mesh difference schemes for singularly perturbed two-point boundary value problems, Math. Comp. 51 (1988), no. 184, 631-657. MR 89d:65073. Zbl 699.65063.

[6] M. K. Kadalbajoo and A. A. Rao, The alternating group explicit (AGE) method for singularly perturbed boundary value problems, Appl. Math. Comput. 68 (1995), no. 2-3, 125142. MR 96a:65114. Zbl 821.65059.

[7] T. R. Lucas and G. W. Reddien, Jr., Some collocation methods for nonlinear boundary value problems, SIAM J. Numer. Anal. 9 (1972), 341-356. MR 46\#8443. Zbl 266.34024.

Dialla Konate: 37 Rue de la RéPublique, 92800 Puteaux, France

E-mail address: dkonate@wanadoo.fr 


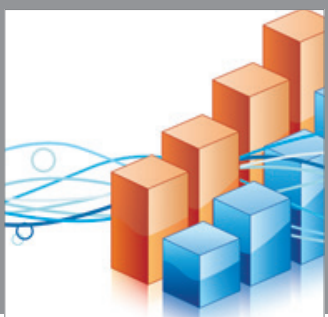

Advances in

Operations Research

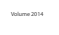

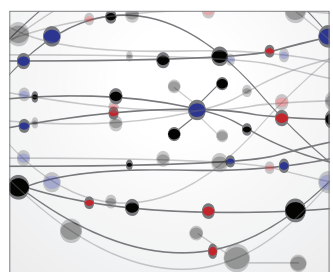

\section{The Scientific} World Journal
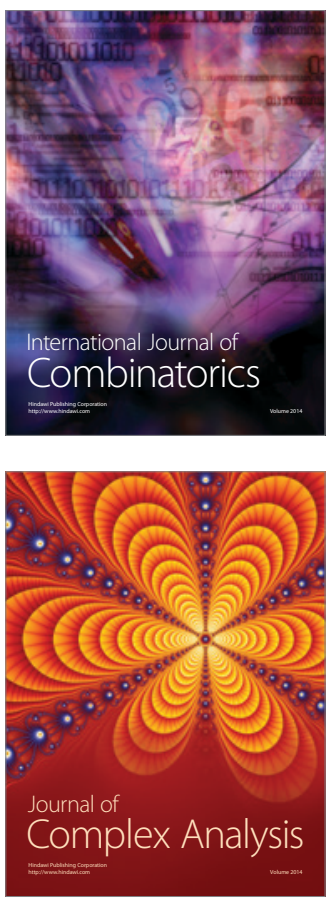

International Journal of

Mathematics and

Mathematical

Sciences
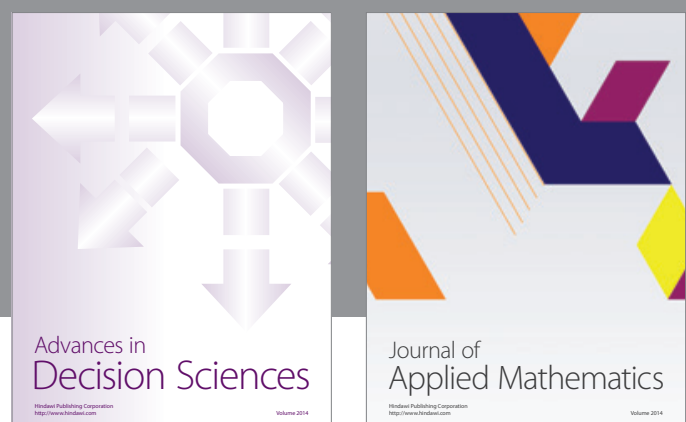

Journal of

Applied Mathematics
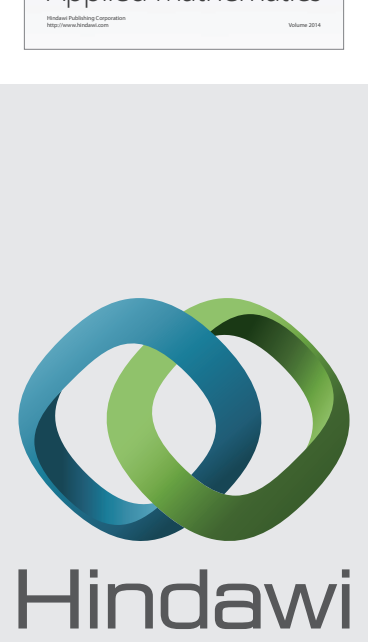

Submit your manuscripts at http://www.hindawi.com
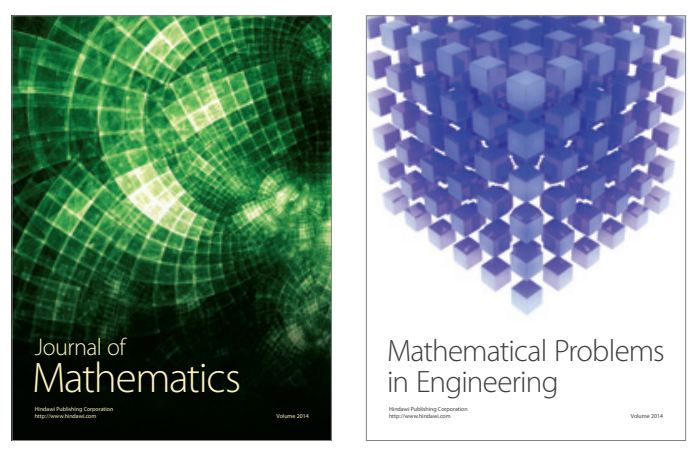

Mathematical Problems in Engineering
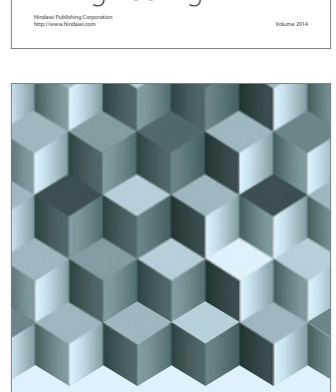

Journal of

Function Spaces
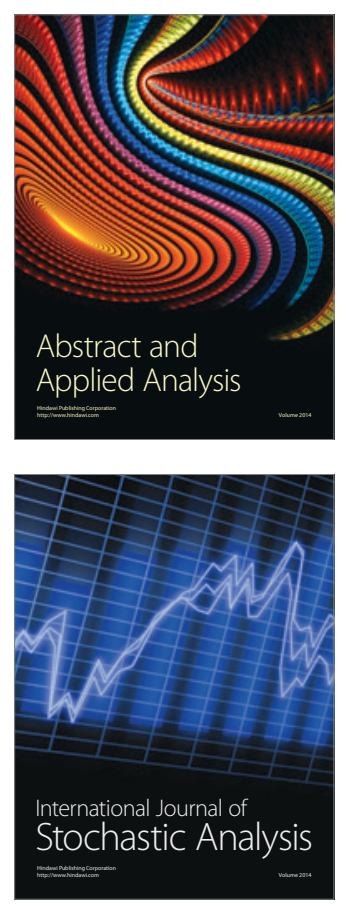

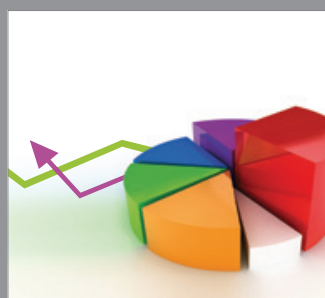

ournal of

Probability and Statistics

Promensencen
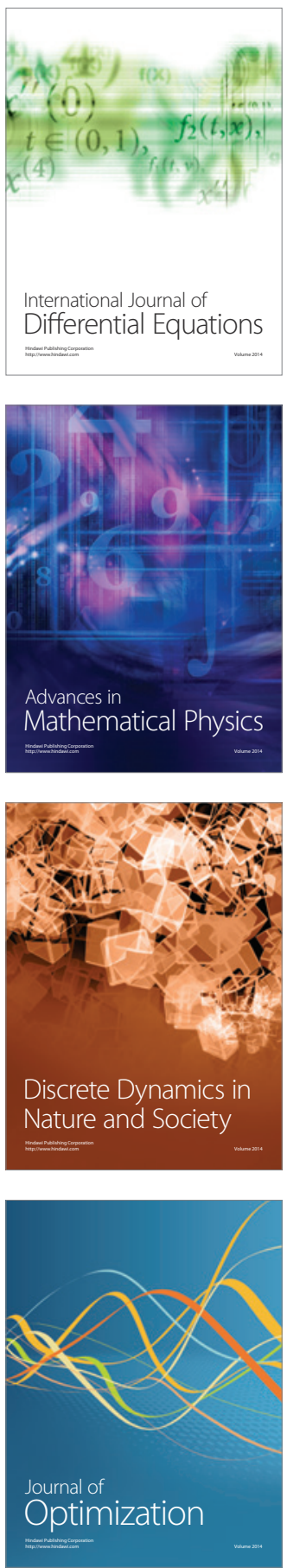